\title{
In Vivo Selection of Intermediately- and Highly- Malignant Variants of Triple-negative Breast Cancer in Orthotopic Nude Mouse Models
}

\author{
SHUYA YANO ${ }^{1,2,3}$, KIYOTO TAKEHARA ${ }^{1,2,3}$, HIROYUKI KISHIMOTO $^{3}$, HIROSHI TAZAWA $^{4}$, YASUO URATA ${ }^{5}$, \\ SHUNSUKE KAGAWA ${ }^{3}$, MICHAEL BOUVET ${ }^{2}$, TOSHIYOSHI FUJIWARA ${ }^{3}$ and ROBERT M. HOFFMAN ${ }^{1,2}$ \\ ${ }^{1}$ AntiCancer, Inc., San Diego, CA, U.S.A.; \\ ${ }^{2}$ Department of Surgery, University of California San Diego, CA, U.S.A.; \\ ${ }^{3}$ Department of Gastroenterological Surgery, Okayama University, Graduate School of Medicine, \\ Dentistry and Pharmaceutical Sciences, Okayama, Japan; \\ ${ }^{4}$ Center for Innovative Clinical Medicine, Okayama University Hospital, Okayama, Japan; \\ ${ }^{5}$ Oncolys BioPharm Inc., Tokyo, Japan
}

\begin{abstract}
Aim: Triple-negative breast cancer (TNBC), defined by the absence of receptors for estrogen, progesterone and human epithelial receptor 2 (HER2), is a recalcitrant disease in need of effective therapy. We previously isolated very-highly metastatic variants of the TNBC cell line MDA-MB-231 using serial orthotopic implantation of $M D A-M B-231$ human breast cancer cells in nude mice. Materials and Methods: MDA-MB231 cells expressing red fluorescent protein (MDA-MB-231$R F P)\left(1 \times 10^{7}\right.$ cells/site $)$ were initially injected subcutaneously in the flank of nude mice. After the subcutaneous tumors grew, they were harvested and cut into small pieces for orthotopic implantation into the right lower mammary gland. After the orthotopic tumors grew, they were resected and cut into small pieces and orthotopically re-implanted into the mammary gland of nude mice. The tumors grew and metastasized to lymph nodes. The lymph node metastases were harvested and cut into small pieces and orthotopically re-implanted into the mammary gland of nude mice. After the orthotopic tumors grew, the tumor was removed leaving residual cancer cells, which grew and
\end{abstract}

This article is freely accessible online.

Correspondence to: Robert M. Hoffman, Ph.D., AntiCancer, Inc., 7917 Ostrow Street, San Diego, CA 92111, U.S.A. Tel: +1 8586542555, Fax: +1 8582684175, e-mail: all@anticancer.com or Toshiyoshi Fujiwara, MD, Ph.D., Department of Gastroenterological Surgery, Okayama University Graduate School of Medicine, Dentistry, and Pharmaceutical Sciences, 2-5-1 Shikata-cho, Kita-ku, Okayama 700-8558, Japan. Tel: +81 862357255, Fax: +81 862218775, e-mail: toshi_f@md.okayama-u.ac.jp

Key Words: Triple-negative breast cancer, TNBC, MDA-MB-231, nude mice, orthotopic, red fluorescent protein, RFP, in vivo selection. metastasized to lymph nodes. The lymph node metastases were harvested, cut into pieces and orthotopically re-implanted into the mammary gland of nude mice for three or seven cycles in order to isolate intermediately, or highly-metastatic variants, respectively. Results: The degree of malignancy of isolated variants of MDA-MB-231 depends on the extent of orthotopic transplantation. Serial transplantation resulted in MDA-MB231-RFP which significantly produced larger tumors compared with the parental MDA-MB-231-RFP. Serial orthotopic implantation for three cycles resulted in intermediatelymetastatic variants of MDA-MB-231-RFP. MDA-MB-231-RFP serially orthotopically transplanted seven times significantly metastasized more to lymph nodes compared with parental MDA-MB-231-RFP cells and the intermediately-metastatic variant. The highly-metastatic variant MDA-MB-231-RFP cells significantly metastasized to the lung to a greater extent compared with parental MDA-MB-231-RFP and intermediate variants of MDA-MB-231-RFP. Conclusion: These results suggest that the number of serial orthotopic transplantations of MDA-MB-231-RFP correlates positively with tumor aggressiveness, and the intermediately-and highly-metastatic variants can serve as models of metastatic progression of TNBC.

Triple-negative breast cancer (TNBC), defined by the absence of receptors for estrogen, progesterone and human epithelial receptor 2 (HER2), is a recalcitrant disease in need of effective therapy $(1,2)$.

We previously developed a highly-invasive, TNBC variant using serial orthotopic implantation of MDA-MB-231 human breast cancer expressing red fluorescent protein (MDA-MB231-RFP) in nude mice (3). MDA-MB-231-RFP was implanted orthotopically into the right lower mammary gland. After the orthotopic tumors grew, they were re- 
implanted into the mammary gland of nude mice. The tumors grew and metastasized to lymph nodes. The lymphnode metastases were harvested and orthotopically reimplanted into the mammary gland of nude mice. After the orthotopic tumors grew, the tumor was resected leaving residual cancer cells, which grew and metastasized to lymph nodes. The lymph-node metastases were harvested, cut and orthotopically re-implanted into the mammary gland of nude mice for additional cycles and then isolated. The isolated variant was highly invasive in the mammary gland and metastasized to lymph nodes in 10 out of 12 mice compared to two out of 12 of the parental cell line (3).

The present report describes isolation of an intermediately-aggressive variant of MDA-MB-231-RFP and compares it to the highly-aggressive model, as well as to the parental cell line, showing that the set of variants can serve as models of metastatic progression of TNBC.

\section{Materials and Methods}

Cell culture. Parental and metastatic variants of the TNBC MDAMB-231P-RFP cell line were maintained and cultured in Dulbecco's modified Eagle's medium (DMEM) on plastic with $10 \%$ fetal bovine serum and $5 \%$ penicillin/streptomycin.

Mice. Athymic nude mice (AntiCancer Inc., San Diego, CA, USA) were kept in a barrier facility under HEPA filtration. Mice were fed with autoclaved laboratory rodent diet (Tecklad LM-485, Envigo, Indianapolis, IN, USA). All animal studies were conducted in accordance with the principles and procedures outlined in the National Institutes of Health Guide for the Care and Use of Laboratory Animals under assurance A3873-01.

Establishment of intermediately- and highly-metastatic variants by serial orthotopic implantation. Initially, MDA-MB-231-RFP cells $\left(1 \times 10^{7}\right.$ cells/site $)$ were injected subcutaneously into the flank of 5 nude mice. For orthotopic transplantation, $7-\mathrm{mm}^{3}$ fragments of the harvested subcutaneous tumor were grafted into a mammary gland of 5 nude mice. After growth, the tumor was resected. Residual cancer cells grew into a tumor which was harvested and divided into fragments and re-implanted into a mammary gland. After the tumor metastasized to lymph nodes, it was harvested and divided into fragments and re-implanted into mammary gland. After growth, the orthotopic tumor was resected. The residual cancer cells formed primary and metastatic tumors. The metastasis was harvested and divided into fragments re-implanted into the mammary gland. Highly-metastatic MDA-MB-231-RFP tumors were developed after seven orthotopic transplantations described above. Intermediatelymetastatic variants of MDA-MB-231-RFP were isolated by serial orthotopic transplantation three times (3-6).

In vivo whole-body/whole-tumor imaging. The OV100 small animal imaging system (Olympus Corp., Tokyo, Japan), was used. The OV100 contains an MT-20 light source (Olympus Biosystems, Planegg, Germany) and DP70 CCD camera (Olympus), for whole body, as well as subcellular imaging in live mice (7-9). The optics of the OV100 have been specially developed for macroimaging as well as microimaging with high light-gathering capacity. Four individually optimized objective lenses, parcentered and parfocal, provide a 105 -fold magnification range. High-resolution images were captured directly on a PC (Fujitsu Siemens, Munich, Germany). Images were processed for contrast and brightness and analyzed with the use of Paint Shop Pro 8 and CellR (10-15).

Confocal laser scanning microscopy (CLSM) was performed using the FV1000 (Olympus Corp.) with 2-laser diodes (473 nm and $559 \mathrm{~nm})$. A 4x (0.20 numerical aperture immersion) objective lens and $20 \times$ (0.95 numerical aperture immersion) objective lens (Olympus) were used. Scanning and image acquisition were controlled by Fluoview software (Olympus) (16).

Statistical analysis. Data for fluorescence intensity (Figure 1C, 2C) are shown as means \pm standard deviation (SD). For comparison between two groups, significant differences were determined using the Student's $t$-test. $P$-values of less than 0.05 were considered significant.

\section{Results and Discussion}

The degree of malignancy of isolated variants of MDA-MB231-RFP depended on the extent of orthotopic transplantation. Intermediately- and highly-metastatic variants of MDA-MB-231-RFP were developed by serial orthotopic transplantation in the mammary gland using lymph-node metastasis. Serial transplantation resulted in MDA-MB-231-RFP cells which produced significantly larger tumors compared with parental MDA-MB-231-RFP cells. Serial orthotopic implantation three times resulted in

Figure 1. Selection of weakly-, intermediately and highly-metastatic variants of MDA-MB-231-RFP triple-negative breast cancer: primary growth and lymph-node metastasis. For orthotopic transplantation, fragments of MDA-MB-231P-RFP tumors were used for grafting into mammary glands of nude mice. After the orthotopic tumor was resected, residual cancer cells were allowed to grow into tumors and metastasis which were harvested and re-implanted into mammary glands of other nude mice. After metastasis to the lymph nodes, the metastatic tumors were harvested and reimplanted into mammary glands of nude mice. Intermediately-metastatic MDA-MB-231-RFP variants were developed after three serial orthotopic transplantations of lymph-node metastasis tissue. Highly-metastatic MDA-MB-231-RFP variants were isolated after seven serial orthotopic transplantations of lymph-node tissue. A: Representative whole-body images of primary tumors from parental, intermediately-metastatic and highlymetastatic MDA-MB-231-RFP tumors. B: Representative images of hemotoxylin and eosin (H\&E)-stained tissue sections from primary tumors from parental, intermediately-metastatic and highly-metastatic MDA-MB-231-RFP tumors. Bar=200 $\mu$ m (upper row). Bar=50 $\mu \mathrm{m}$ (lower row). C: Bar graphs show the comparison of fluorescence intensity from primary tumors (left) and lymphatic metastases (right) in mice with parental, intermediately-metastatic and highly-metastatic MDA-MB-231-RFP variant tumors. Fluorescence imaging was with the OV100 (10-15). 


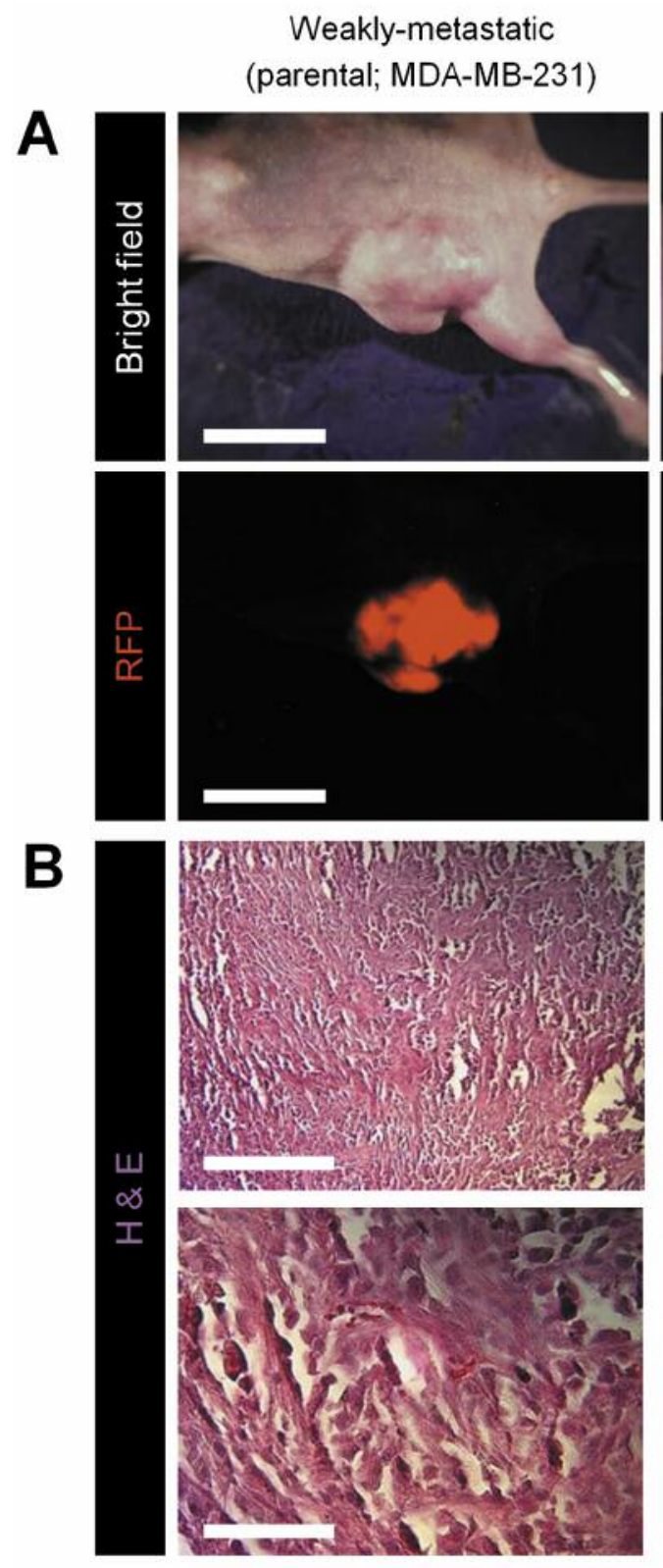

C

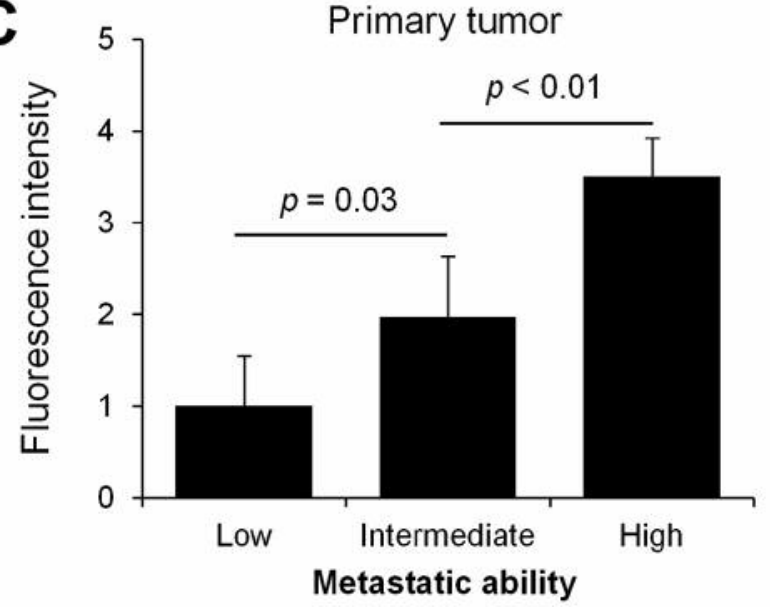

Intermediately-metastatic

(MDA-MB-231)
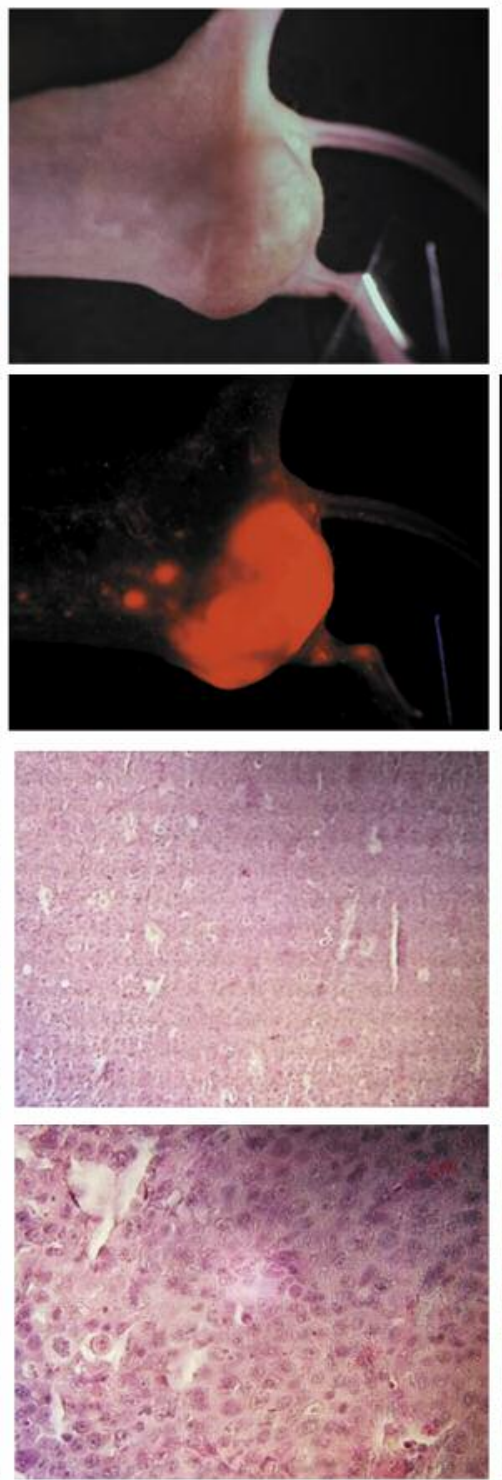

Highly-metastatic

(MDA-MB-231)
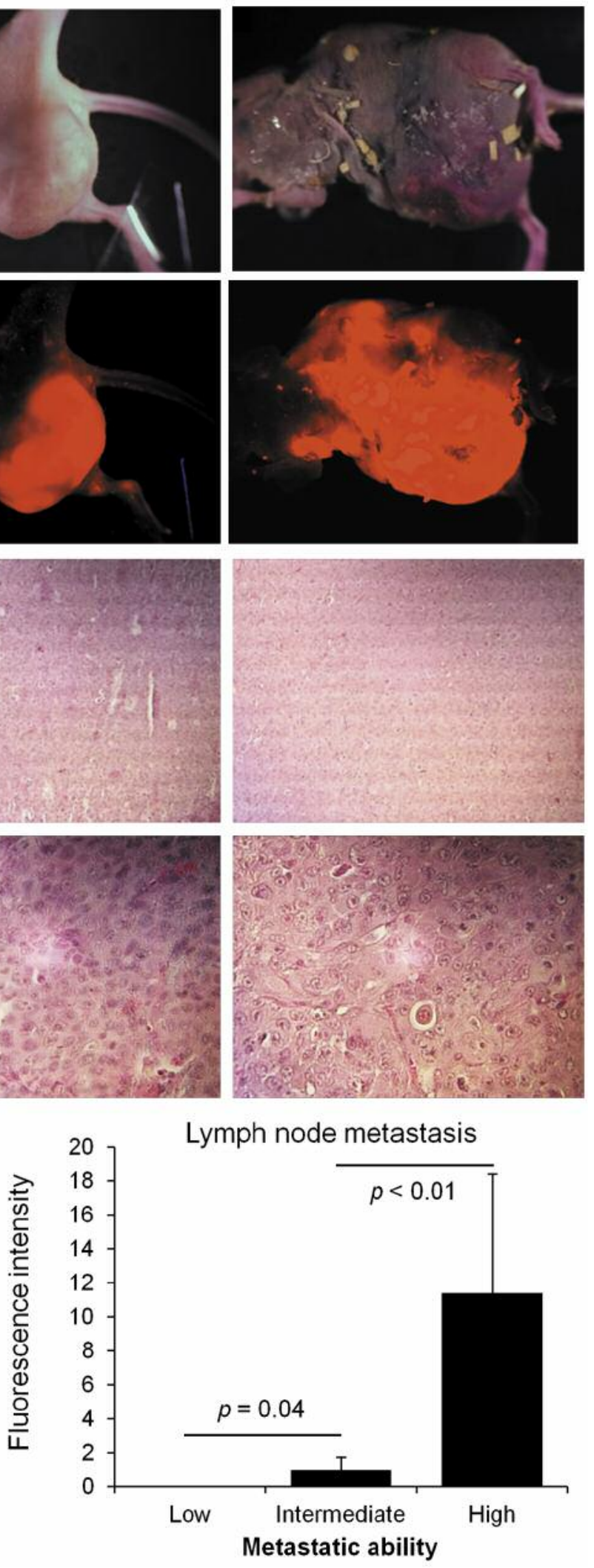
A Weakly-metastatic (parental; MDA-MB-231)
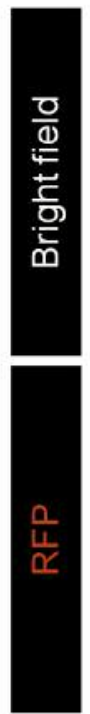

B
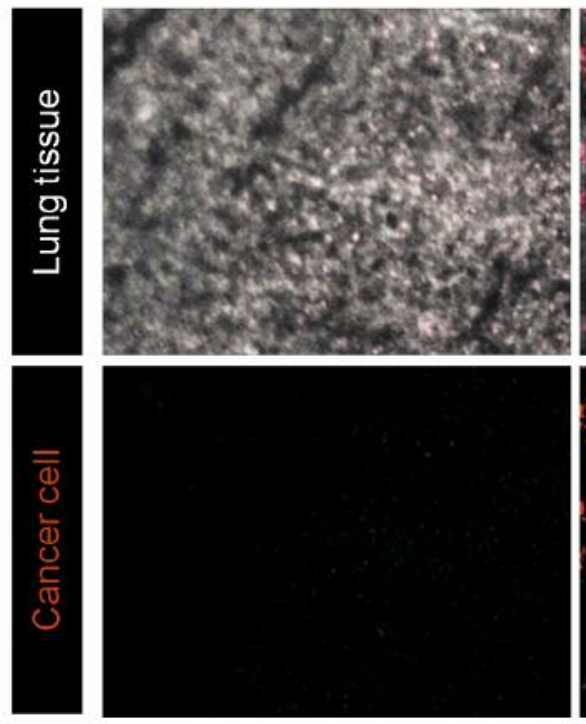

C

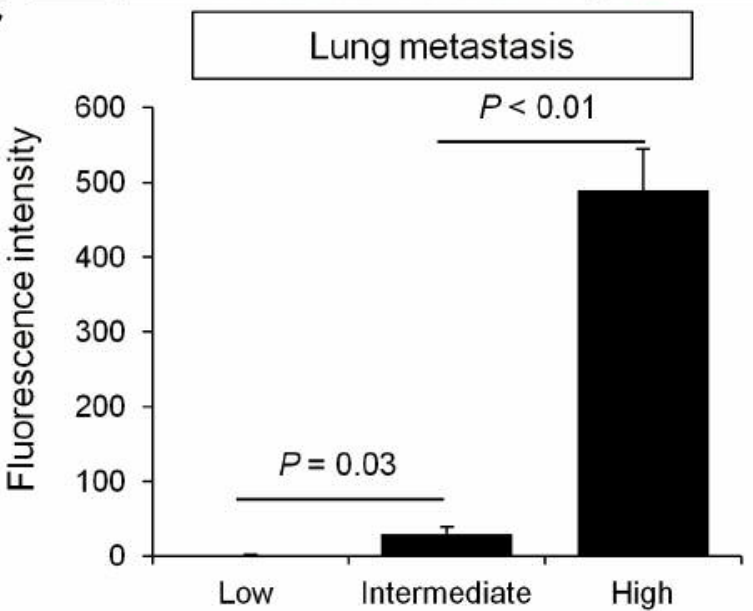

Intermediately-metastatic

(MDA-MB-2311)
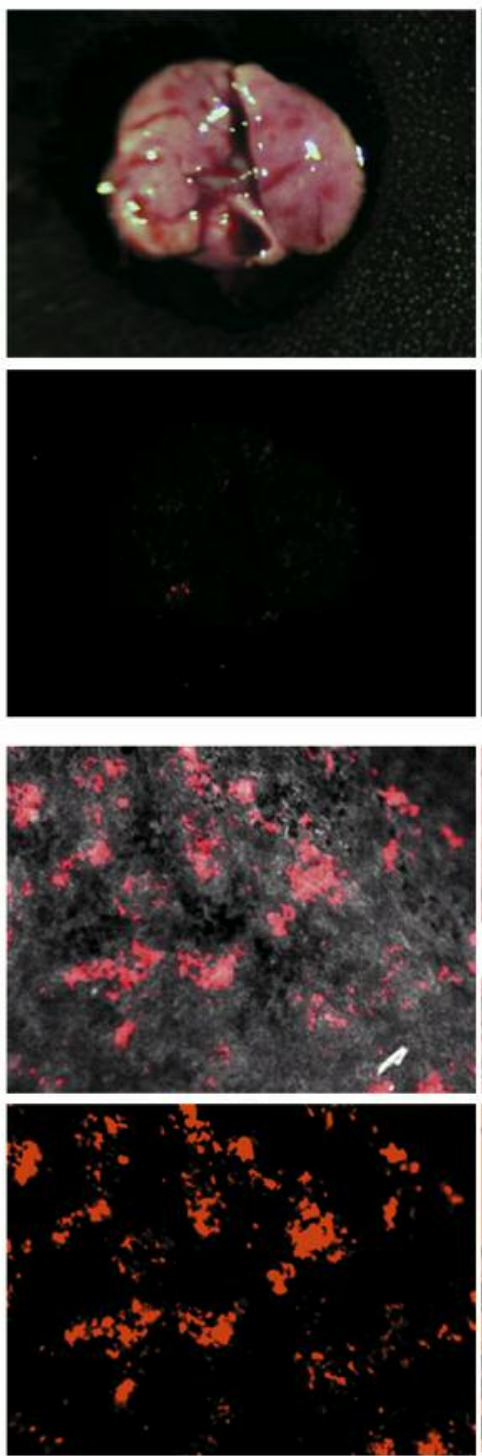

Highly-metastatic

(MDA-MB-231)
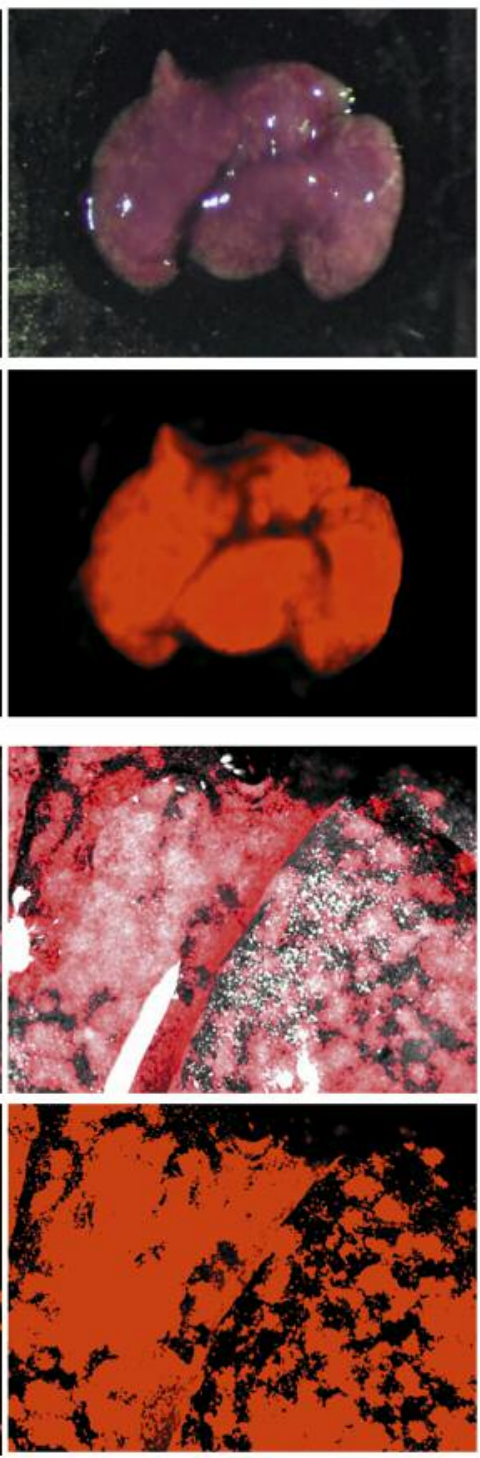

Figure 2. Lung metastasis from parental, intermediately-metastatic and highly-metastatic MDA-MB-231-RFP. A: Representative images of whole lungs from mice with parental, intermediately-metastatic and highlymetastatic MDA-MB-231-RFP tumors. B: Representative single-cell images of lung metastasis from parental, intermediately- metastatic and highly-metastatic MDA-MB-231-RFP tumors. C: Bar graphs show the comparison of fluorescence intensity from lung metastasis in mice with parental, intermediately metastatic and highly-metastatic MDA-MB-231RFP tumors. A: Imaged with the OV100. B: Imaged with the FV1000. 
intermediately-metastatic variants of MDA-MB-231-RFP. The highly-metastatic MDA-MB-231-RFP variant serially transplanted seven times grew significantly faster at the primary site (Figure 1A and $\mathrm{B}$ ), and metastasized more to lymph nodes compared with parental MDA-MB-231-RFP and the intermediately-metastatic variant (Figure 1C). The highly-metastatic MDA-MB-231-RFP variant significantly metastasized to the lung to a greater extent compared with parental MDA-MB-231-RFP and the intermediately metastatic variant of MDA-MB-231-RFP (Figure 2A-C).

These results suggest that the number of serial orthotopic transplantations of MDA-MB-231-RFP positively correlates with aggressiveness and the intermediately and highly metastatic variants can serve as models of metastatic progression of TNBC.

\section{Financial Disclosure}

This study was supported in part by the National Cancer Institute grant CA 132971 and CA142669. This study was also supported in part by grants from the Ministry of Health, Labour, and Welfare, Japan (to T. Fujiwara; No. 10103827, No. 13801426, No. 14525167) and grants from the Ministry of Education, Culture, Sports, Science and Technology, Japan (to T. Fujiwara; No. 25293283).

\section{Conflicts of Interest}

There are no conflicts of interest.

\section{Acknowledgements}

This article is dedicated to the memory of A.R. Moossa, MD, and Sun Lee, MD.

\section{References}

1 Devita VT Jr., Lawrence TS and Rosenberg SA (eds.): Devita, Hellman and Rosenberg's Cancer: Principles and Practice of Oncology, 10th Edition, Wolters Kluwer Health (U.S.A.), 2015.

2 Foulkes WD, Smith IE and Reis-Filho JS: Triple-negative breast cancer. N Engl J Med 363: 1938-1948, 2010.

3 Yano S, Takehara K, Miwa S, Kishimoto H, Tazawa H, Urata Y, Kagawa S, Bouvet M, Fujiwara T and Hoffman RM: In vivo isolation of a highly-aggressive variant of triple-negative human breast cancer variant MDA-MB-231 using serial orthotopic transplantation. Anticancer Res 36: 3817-3820, 2016.
4 Hoffman RM: Patient-derived orthotopic xenografts: better mimic of metastasis than subcutaneous xenografts. Nat Rev Cancer 15: 451-452, 2015.

5 Hoffman RM: Orthotopic metastatic mouse models for anticancer drug discovery and evaluation: a bridge to the clinic. Investigational New Drugs 17: 343-359, 1999.

6 Li X-M, Wang J-W, An Z, Yang M, Baranov E, Jiang P, Sun F$\mathrm{X}$, Moossa AR and Hoffman RM: Optically imageable metastatic model of human breast cancer. Clinical \& Experimental Metastasis 19: 347-350, 2002.

7 Hoffman RM and Yang M: Subcellular imaging in the live mouse. Nat Protocol 1: 775-782, 2006.

8 Hoffman RM and Yang M: Color-coded fluorescence imaging of tumor-host interactions. Nat Protocol 1: 928-935, 2006.

9 Hoffman RM and Yang M: Whole-body imaging with fluorescent proteins. Nat Protocol 1: 1429-1438, 2006.

10 Yamauchi K, Yang M, Jiang P, Xu M, Yamamoto N, Tsuchiya H, Tomita K, Moossa AR, Bouvet M and Hoffman RM: Development of real-time subcellular dynamic multicolor imaging of cancer cell trafficking in live mice with a variable magnification whole-mouse imaging system. Cancer Res 66: 4208-4214, 2006.

11 Hoffman RM: The multiple uses of fluorescent proteins to visualize cancer in vivo. Nat Rev Cancer 5: 796-806, 2005.

12 Yang M, Baranov E, Jiang P, Sun F-X, Li X-M, Li L, Hasegawa S, Bouvet M, Al-Tuwaijri M, Chishima T, Shimada H, Moossa AR, Penman S and Hoffman RM: Whole-body optical imaging of green fluorescent protein-expressing tumors and metastases. Proc Natl Acad Sci USA 97: 1206-1211, 2000.

13 Hoffman RM: Application of GFP imaging in cancer. Laboratory Investigation 95: 432-452, 2015.

14 Hoffman RM (ed.): In Vivo Cellular Imaging Using Fluorescent Proteins: Methods and Protocols. Methods in Molecular Biology, Vol. 872. Walker, JM., series ed. Humana Press (Springer Science+ Business Media New York), 2012.

15. Hayashi K, Jiang P, Yamauchi K, Yamamoto N, Tsuchiya H, Tomita K, Moossa AR, Bouvet M and Hoffman RM: Real-time imaging of tumor-cell shedding and trafficking in lymphatic channels. Cancer Res 67: 8223-8228, 2007.

16. Uchugonova A, Duong J, Zhang N, König K and Hoffman RM: The bulge area is the origin of nestin-expressing pluripotent stem cells of the hair follicle. J Cell Biochem 112: 2046-2050, 2011.

Received September 23, 2016 Revised October 21, 2016 Accepted October 24, 2016 